\title{
Dilong: Role in Peripheral Nerve Regeneration
}

\author{
Yung-Ming Chang, ${ }^{1,2}$ Wei-Yi Chi, ${ }^{3,4}$ Tung-Yuan Lai, ${ }^{4}$ Yueh-Sheng Chen, ${ }^{1}$ Fuu-Jen Tsai, ${ }^{5}$ \\ Chang-Hai Tsai, ${ }^{6}$ Wei-Wen Kuo, ${ }^{7}$ Yi-Chang Cheng, ${ }^{8}$ Chien-Chung Lin, ${ }^{9}$ \\ and Chih-Yang Huang ${ }^{5,10,11}$
}

\author{
${ }^{1}$ School of Chinese Medicine, China Medical University, Taichung, Taiwan \\ ${ }^{2}$ The Pre-Clinical Medicine College, Nanjing University of Traditional Chinese Medicine, Nanjing 210029 Jiangsu, China \\ ${ }^{3}$ Drexel University, School of Nursing and Health Professions, Department of Physical Therapy and Rehabilitation Sciences, \\ Philadelphia, PA, USA \\ ${ }^{4}$ School of Post-Baccalaureate Chinese Medicine, China Medical University, Taichung 404, Taiwan \\ ${ }^{5}$ Graduate Institute of Chinese Medical Science, China Medical University, Taichung 404, Taiwan \\ ${ }^{6}$ Department of Healthcare Administration, Asia University, Taichung, Taiwan \\ ${ }^{7}$ Department of Biological Science and Technology, China Medical University, Taichung, Taiwan \\ ${ }^{8}$ Emergency Department, Taichung Veterans General Hospital, Taiwan \\ ${ }^{9}$ Orthopaedic Department, Armed Forces General Hospital, Taichung, Taiwan \\ ${ }^{10}$ Graduate Institute of Basic Medical Science, China Medical University, Taichung 404, Taiwan \\ ${ }^{11}$ Department of Health and Nutrition Biotechnology, Asia University, Taichung 413, Taiwan
}

Correspondence should be addressed to Chih-Yang Huang, cyhuang@mail.cmu.edu.tw

Received 21 April 2010; Accepted 25 May 2010

Copyright (C) 2011 Yung-Ming Chang et al. This is an open access article distributed under the Creative Commons Attribution License, which permits unrestricted use, distribution, and reproduction in any medium, provided the original work is properly cited.

Dilong, also known as earthworm, has been widely used in traditional Chinese medicine (TCM) for thousands of years. Schwann cell migration and proliferation are critical for the regeneration of injured nerves and Schwann cells provide an essentially supportive role for neuron regeneration. However, the molecular mechanisms of migration and proliferation induced by dilongs in Schwann cells remain unclear. Here, we discuss the molecular mechanisms that includes (i) migration signaling, MAPKs (mitogen-activated protein kinases), mediated PAs and MMP2/9 pathway; (ii) survival and proliferative signaling, IGF-I (insulinlike growth factor-I)-mediated PI3K/Akt pathways and (iii) cell cycle regulation. Dilong stimulate RSC96 cell proliferation and migration. It can induce phosphorylation of ERK1/2 and p38, but not JNK, and activate the downstream signaling expression of PAs (plasminogen activators) and MMPs (matrix metalloproteinases) in a time-dependent manner. In addition, Dilong stimulated ERK1/2 and p38 phosphorylation was attenuated by pretreatment with chemical inhibitors (U0126 and SB203580), and small interfering ERK1/2 and p38 RNA, resulting in migration and uPA-related signal pathway inhibition. Dilong also induces the phosphorylation of IGF-I-mediated PI3K/Akt pathway, activates protein expression of PCNA (proliferating cell nuclear antigen) and cell cycle regulatory proteins (cyclin D1, cyclin E and cyclin A) in a time-dependent manner. In addition, it accelerates $\mathrm{G}_{1}$ phase progression with earlier S-phase entry and significant numbers of cells entered the S-phase. The siRNA-mediated knockdown of PI3K that significantly reduces PI3K protein expression levels, resulting in $\mathrm{Bcl}_{2}$ survival factor reduction, revealing a marked blockage of $G_{1}$ to $S$ transition in proliferating cells. These results reveal the unknown RSC96 cell migration and proliferation mechanism induced by dilong, which find use as a new medicine for nerve regeneration.

\section{Introduction}

1.1. Regeneration of Nerves. Nerve regeneration is a complex phenomenon that has interested scientists for many years. Neurons can be separated into central and peripheral nervous systems (PNSs), which have different anatomical structures and regenerative ability. In mammals, the central neurons without a myelin sheath are difficult to regenerate. In contrast to the central nervous system, the PNS with a myelin sheath exhibit easier regrowth [1]. Regrowth ability results from intrinsic neuronal activities and surrounding non-neuronal properties in which Schwann cells provide 
an essentially supportive activity for neuron regeneration. Schwann cells are the supporting cells of the PNS and can differentiate into the myelin sheath of the PNS and proliferate and migrate into the distal end of the injured nerve area [2]. Moreover, Schwann cell migration, which also occurs at the proximal end of the injured area, provides a guide for regenerating axons by interacting with nerve fibers or basal lamina [3]. Since Schwann cell migration is critical for axonal elongation and remyelination of injured nerves $[3,4]$, those factors that regulate Schwann cell migration have been widely investigated. Peripheral nerve injury locally activates Schwann cells and macrophages to synthesize a cocktail of neurotrophic factors, adhesion molecules, cytokines and growth-promoting surface molecules $[5,6]$. However, the mechanisms of action of these regulating factors on Schwann cell migration, proliferation and signal transformation remain unclear.

\subsection{Pathways that Play a Role in Cellular Proliferation and} Migration. The mitogen-activated protein kinase (MAPK) family plays an essential role in inducing cell proliferation [7] and migration [8]. Extracellular signal-regulated protein kinase (ERK) that belongs to MAPK family has been studied extensively [9]. Results reveal that ERK is related to migration of various cell types, including fibroblasts and carcinoma cells $[10,11]$, but not in Schwann cells. Recently, several studies found that after nerve injury, the increased activation of ERK [12] phosphorylation promotes neurite outgrowth [13]. Interestingly, to promote migration, growth cones at the tip of an axon secrete proteases that are thought to dissolve cell-cell and cell-matrix adhesions during peripheral nerve regeneration. These proteases include the plasminogen activators (PAs), tissue PA (tPA) and urokinase PA (uPA) and their substrate, plasminogen [14]. Many experiments have determined that after injury, a rapid increase of tissue PA expression has been observed in neurons [14, 15]. Tissue PA or uro kinase PA activates plasmin that consequently activates MMP-9 and MMP-2 [16]. It has been shown that the lack of plasminogen activators affects MMP-9 and MMP-2 activity [17]. However, little is known about Schwann cell migration using MEK/ERK signaling pathways to active PAs and MMPs. In addition, accumulating evidence has also indicated that c-Jun NH2-terminal kinase (JNK) and $\mathrm{p} 38$, the other two members of the MAPK super family, have the affection on cell migration regulation [10]. To promote migration in cells, the expression of matrixdegrading proteolytic enzymes (PAs and MMPs) could be regulated by JNK [18] and p38 [19] signal transduction pathways.

\section{Role of Growth Factors}

2.1. Insulin-Like Growth Factors. Insulin-like growth factor-I (IGF-I) is a polypeptide hormone synthesized by proliferating Schwann cells [20]. The secretion of IGF-I is controlled by the growth hormone [21]. In response, IGF-I stimulates the growth and differentiation of fetal neurons [22] and increases neurite sprouting and outgrowth in vitro $[23,24]$. Interestingly, IGF-I not only stimulates proliferation but also promotes survival in several cell types. It can rescue Schwann cells from apoptosis via PI3-K signaling, which is upstream from caspase activation [25]. In vivo, the signal cascade for early upregulation of IGF-I has been shown to promote retinal ganglion cells (RGCs) survival and axonal regeneration through the $\mathrm{PI} 3 \mathrm{~K} / \mathrm{Akt}$ system after optic nerve injury in goldfish [26].

2.2. PI3K/Akt System and Peripheral Nerve Regulation. Many studies have investigated the effects of the PI3K/Akt system on peripheral nerve regeneration. IGF-I functions as a progression factor in the cell cycle [27], promoting $\mathrm{G}_{1} / \mathrm{S}$ cell cycle progression via the phosphatidylinositol 3kinase/serine-threonine kinase (PI3K/Akt) pathway, resulting in DNA synthesis and cell proliferation [28]. This hormone protects neurons in the PNS from apoptosis by activating the PI3K/Akt pathway, which in turn phosphoralates Bad and activates $\mathrm{Bcl}_{2}$, an anti-apoptotic protein that interferes with the activation of caspases [26, 29, 30]. Furthermore, it has been suggested that the inhibition of PI3K activation can completely block Schwann cell proliferation and survival [31]. Generally, these data strongly indicate that IGF-I is an important molecule for controlling regeneration after nerve injury. Therefore, IGF-I has been used as a therapeutic target for the treatment of peripheral nerve injury and motor neuron diseases [32]. Insulin-like growth factor I (IGF-I) is currently in clinical trials for treatment of amyotrophic lateral sclerosis (ALS) based on its neuroprotective effect on motor neurons [33].

\section{Traditional Chinese Medicine}

3.1. Is There a Role for Dilong? The pharmacology and clinical application of traditional Chinese medicine has been well documented for several thousand years. Recently, biomedical material science combined with Chinese herbal medicine has been applied to analyses of nerve regeneration. Several Chinese medicines have been identified as enhancing neuron regeneration. In Tsai's study, ginsenoside Rb1 (GRb1) filled into a silicon rubber chamber can bridge a $15-\mathrm{mm}$ gap in injured rat sciatic nerves [34]. Therefore, the herbal medicine has a good potential for treating injured nerves. The earthworms, also called "dilong" in Chinese, are a widely used Chinese herbal medicine [35]. Extracting medicinal compounds from dilong has traditionally been practiced by indigenous people throughout the world, more particularly in Asia [36]. The potential treatment effects of earthworms may come from their dense soil-based nutritional content [37].

Bu Yang Huan Wu Tang (BYHWT), a Chinese herb complex prescription, has been applied for treating the sequelae of stroke, eye and mouth distortion, stiffness in the tongue and aphasia, as well as atrophy and paralysis of lower limbs. BYHWT consists of Dilong (Earthworm), Chi Shao (Peony Red), Tao Ren (Persica), Hong Hua (Carthamus), Dang Gui (Chinese Angelica Root), Huang Qi (Astragalus) and Chuan Xiong (Cnidium). It has been shown that BYHWT can increase the level of NO in cerebral infarct rats and reduce the area of cerebral infarct [38]. Furthermore, several research 


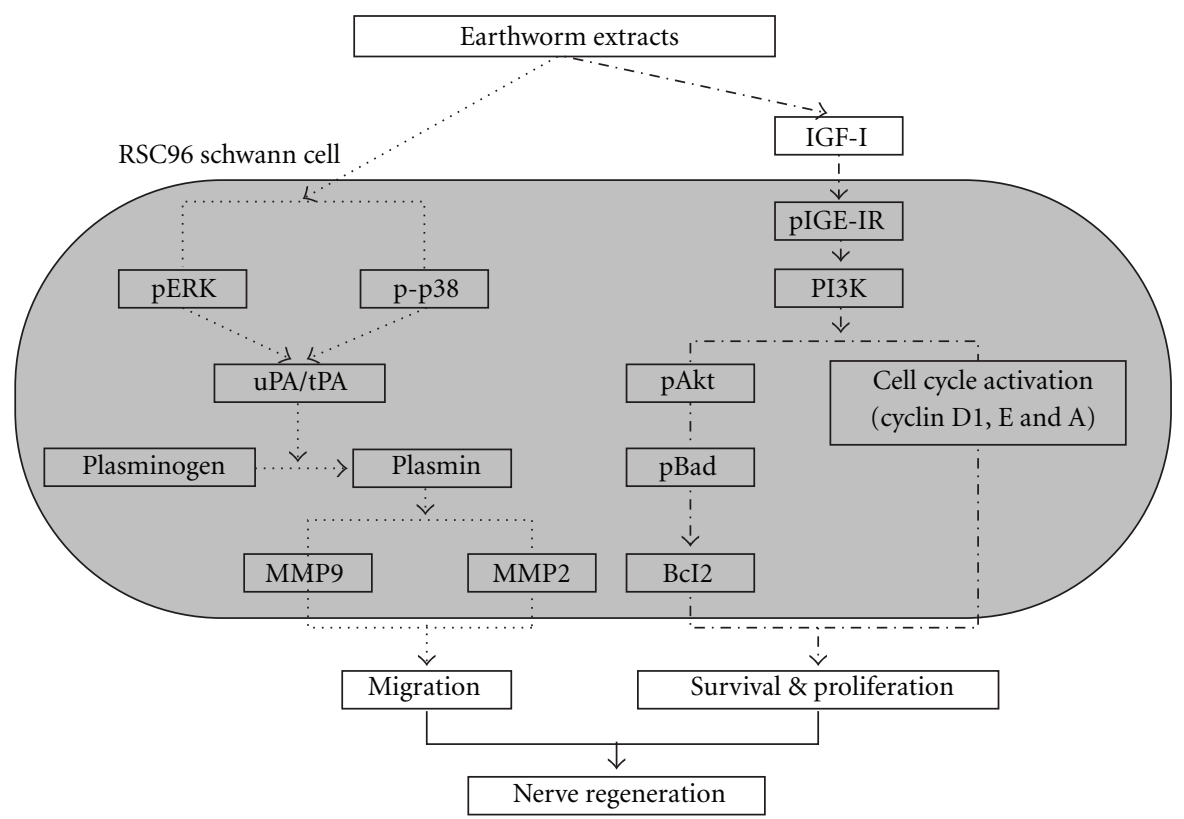

FIGURE 1: Schematic model of migrative survival and proliferative effects of Dilong extract on Schwann cell.

results indicate that BYHWT may promote the repair and regeneration of neurons and injured nerve fibers [39, 40]. After administered BYHWT for 4 weeks, the conduction velocity of pulses in the newly regenerative nerve of the treatment group was significantly faster than that of the control group. The blood vessel area around the regenerative nerves in the treatment group was also considerably larger. These results provide many information for further studies on the role of Dilong in nerve regeneration.

3.2. Evidence for Diverse Role of Dilong. Previous studies of dilong have shown its antimicrobial [41], hepatoprotective [42], anticancer and scar wound-healing characteristics [35]. The anti-inflammatory activity together with anti-oxidant properties may be due to the high polyphenolic content in dilong tissue [42]. Moreover, crude dilong extract has a thrombolytic effect that could significantly promote blood circulation and remove stasis [43]. In healthy human volunteers, orally administered dilong powder increased levels of tissue plasminogen activator and fibrinolytic activity [44]. These results suggest that earthworm powder represents a possible oral thrombolytic agent.

3.3. Hypothetical Role of Dilong in Nerve Regulation. In vivo experiments have also found that a mixed prescription of liquid extracted from dilong more obviously improves peripheral nerve regeneration than icariin [45]. Therefore, many scientists focus on purifying the contents of crude dilong powder. Different contents of dilong extract will be discussed as follows. First, Lumbrokinase extracted from dilong has been used to treat stroke and cardiovascular diseases [41]. Lumbrokinase is a group of proteolytic enzymes [36]. It includes a plasminogen activator and plasmin [46] that serve to activate plasminogen and dissolve fibrin directly
[47]. Second, dilong tissue homogenates have revealed a glycolipoprotein mixture composed of macromolecules referred to as G-90. G-90 possesses several growth factors and also participates in tissue regeneration and wound healing [48]. Further experimental works are needed to more fully characterize the molecules and potential mechanisms of dilong extract involved in peripheral nerve regeneration.

There is still no conclusive explanation for the possible molecular mechanism involved in Schwann cell migrating and proliferating events. Peripheral nerve regeneration requires a permissive environment and activation of the intrinsic growth capacity of neurons. Axon regrowth and remyelination of the regenerated axons by Schwann cells are both essential. Multiple factors including neurotrophic factors, extracellular matrix (ECM) proteins and hormones participate in Schwann cell dedifferentiation, proliferation and remyelination. A schematic model (Figure 1) is proposed to explain the migrative, survival and proliferative effects of dilong extract on Schwann cells. In this review article, we will discuss the peripheral nerve regeneration mechanisms, with particular attention to the proliferation and migration of Schwann cells induced by dilong.

\section{Migration Signaling: MAPKs Mediated PAs and MMP2/9 Pathway}

4.1. MAPK and Schwann Cell Migration. The MAPK family is a crucial regulator of pathways involved in cell proliferation [7] and migration [8]. JNK, p38 and ERK1/2, the members of MAPKs family, play crucial roles in nerve cells migration [10]. The whole MAPKs pathway is illustrated by the dotted line in Figure 1. This study further demonstrated that dilong extract stimulated ERK1/2 and p38, but not JNK activation in a time-dependent manner, leading to Schwann 
cell migration. Dilong-induced Schwann cell motility and phosphorylation of ERK1/2 and p38 were both attenuated by pretreatment with MEK1/2 (U0126) and p38 (SB203580) inhibitors. Transfection with siRNA of MEK1/2 and p38 significantly reduced migration in response to dilong extract in Schwann cells as well. The signaling migration pathway in earthworm-stimulated Schwann cells, inducing the activation of UPA and TPA mediated through the ERK1/2 and p38. To promote migration, cells secrete proteases (PA and uPA) that are thought to degrade matrix molecules and cell adhesion. The ERK1/2 and p38 phosphorylation leads to the expression of uPA and tPA that occurs in a time-dependent manner, during the elevation of MMP9 and MMP2 levels and activity. In addition, the highly expressed uPA in the epidermis of damaged tissue is also regulated by the fibroblast growth factor (FGF-2) that affects MAPK kinase (MEKK-1) and MEKK-1s downstream extracellular signalregulated kinases (ERK1/2) for controlling UPA expression [49]. Another regulating factor is p38. In endothelial cell migration, the p38 MAPK pathway also participates in by regulating uPA expression [50].

4.2. Dilong Extract and Signaling. In Chang's study, results reveal that dilong extract enhances uPA expression directly through the ERK1/2 and p38 signaling pathway [51]. To promote migration, cells secrete proteases that are thought to degrade matrix molecules and cell adhesion. These proteases include tPA and uPA [14]. In contrast to PAs, PAI-1 is thought to be the main inhibitor of the plasminogen activators. Chang's study clearly shows that the phosphorylation of ERK1/2 and p38 accompanies the increased expression of UPA, but PAI-1 expression is gradually decreased. Interestingly, tPA levels reached the maximal early at $2 \mathrm{~h}$, and then began to decline slightly until $20 \mathrm{~h}$. It suggests that the maximum expression of tPA occurred early following $4 \mathrm{~h}$ treatment, because tPA is the main PAs in the nerve to facilitate growth cone movement by digesting extracellular matrices and cell adhesions. Pittman and DiBenedetto [52] reported that the over expressing tPA can regenerate neurites to a greater extent and migrate faster than the control group in complex extracellular matrix. Ulfhammer et al. [53] found that IPA activation could be mediated through p38 pathways, leading to an increase in tPA expression. Chang's experiments further show that SB203580 inhibited p38 phosphorylation and suppressed tPA protein expression in Schwann cells. Thus, tPA activation occurs not only through ERK1/2 activation but also through the p38 signaling pathway.

\section{Proliferative and Survival Signaling: IGF-I-Mediated PI3K/Akt Pathways}

The PI3K/Akt signaling mediated by IGF-I (dot-dashed line in Figure 1) plays an important role in cell proliferation as well as cell survival $[14,28,30,54]$. IGF-I is a polypeptide hormone synthesized by proliferating Schwann cells [20] and it directly induces neurite outgrowth via a PI3K/Akt-dependent mechanism [26, 55]. Moreover, IGFI rescues Schwann cells from apoptosis via PI3K signaling that is upstream from caspase activation [56], and also requires that PI3K/Akt-mediated progression from G1 to $S$ phase of the cell cycle [54]. Conversely, PI3K inhibitors blocked the anti-apoptotic and protective effects of IGF$\mathrm{I}$, demonstrating that PI3K is essential for trophic factorinduced survival of Schwann cells $[30,56]$. The plasma membrane is subsequently phosphorylated by PI3K. This activates the protein kinase Akt and the following steps of cell proliferation. IGF-1 seems to play an important role in controlling brain growth and cell number [57, 58]. IGF1 injection in embryos increased rat cerebral cortex DNA content by $28 \%$, suggesting a role for IGF-1 in control of brain growth. IGF-1 therefore seems to be a mitogen for cortical precursors during embryonic development [28].

\section{Cell-Cycle Regulation}

6.1. IGF-1. IGF-I has also been shown to function as a progression factor in the cell cycle by regulating expression levels [59]. It can promote $G_{1} / S$ cell cycle progression via the phosphatidylinositol 3-kinase/serine-threonine kinase (PI3K/Akt) pathway, which then results in DNA synthesis and cell proliferation. Chang's analysis indicates that treatment with dilong extract induces phosphorylation of the IGF-I-mediated phosphatidylinositol 3-kinase/serinethreonine kinase (PI3K/Akt) pathway, it activates protein expression of cell nuclear antigen (PCNA) in a time dependent manner [60]. Cell cycle progression is tightly regulated by a complex network of cell cycle regulatory molecules, such as cyclins. Proliferating cells pass through several cell cycle checkpoints, such as the $G_{1}$ to $S$ transitions. G1 checkpoint is considered to be the most important one in the replication of DNA and mitosis. The results show that $G_{1}$ transits into the $S$ phase in $12-16 \mathrm{~h}$, and $S$ transits into the $G_{2}$ phase $20 \mathrm{~h}$ after exposure to dilong extract. Strong expression of cyclin D1, cyclin $\mathrm{E}$ and cyclin A occurs in a time-dependent manner. Progression through the first gap phase (G1) requires cyclin $\mathrm{D}$ and cyclin E activity [61]. Expression of these two cyclin proteins orchestrates the progression of cells through $G_{1}$ and into the S-phase of the cell cycle $[62,63]$. Cyclin A is required for DNA replication in the S-phase and in mitosis initiation (M-phase) [63].

6.2. Small Interfering RNA. Small interfering RNA (siRNA)mediated knockdown of PI3K significantly reduced P13K protein expression levels, resulting in $\mathrm{Bcl}_{2}$ reduction of survival factor and a marked blockage of $G_{1}$ to $S$ transition in proliferating cells. These results demonstrate that dilong extract promotes the proliferation and survival of RSC96 cells via IGF-I signaling. The mechanism is primarily dependent on the PI3K protein [51]. Dilong extract promoted DNA replication and growth of RSC96 cells by upregulating the sequential expression of cyclin D1, cyclin E and cyclin $A$, thereby elevating the number of cells in the $S$ phase in a time-dependent manner. Studies on the proliferative action of IGF-I in cultured fibroblasts (BALB/c-3T3) $[64,65]$ and mammary epithelial cells [66] indicate that IGF-I acts to stimulate progression through $G_{1}$ or the $G_{0} / G_{1}$ transition. Chang's data are in agreement with the results of those studies revealing that the cell cycle is not only regulated 
by cyclins but also mediated by IGF-I [51]. Therefore, cell cycle alterations may be critical determinants of the increased proliferation potency-induced dilong extract.

\section{Perspectives on Schwann Cell Migration}

The mechanism in which dilong extract regulates Schwann cell migration, proliferation and survival is investigated in this article. Specific signaling pathways in dilong-stimulated Schwann cells are shown in Figure 1. For thousands of years dilong has been used as a drug for various diseases in China and the Far East [44]. Until now, the function of dilong extract on nerve regeneration is still unclear. The development and regeneration of the PNS is highly dependent on the migration of Schwann cells and the extension of axons toward their distant targets. Recently studies revealed that MAPKs, including JNK, p38 and ERK1/2, play crucial roles in nerve cell migration [10]. Dilong-induced Schwann cell motility and phosphorylation of ERK1/2 and p38 were both attenuated by pretreatment with MEK1/2 (U0126) and p38 (SB203580) inhibitors. In addition, transfection with siRNA of MEK $1 / 2$ and p38 significantly reduces migration in response to dilong extract in Schwann cells. Using inhibitors and siRNA, the migrative effects of dilong extract on Schwann cells were further identified to be ERK1/2 and p38 signaling dependent. These assays help researchers to examine the individual steps in the complex signaling cascades and clearly illustrate effects of direct dilong extract on Schwann cell migration. Dilong extract enhances uPA expression directly through the ERK1/2 and p38 signaling pathway. When cells treated with dilong extract resulted in ERK1/2 and p38 phosphorylation, the expression of uPA and tPA lead to elevated MMP9 and MMP2 levels and activity. Several experiments have indicated that dilong fibrinolytic enzyme acts as a plasminogen activator [67], suggesting a tPA-like function [68]. Another family of proteases, the matrix metalloproteases (MMPs), are also implicated in peripheral nerve regeneration [69], and involved in many cell migration phenomena and produced by many cell types, including neurons [70]. MMPs are secreted as inactive molecules and require activation via other proteases [71]. Plasmin, activated by tPA or uPA, can activate MMP-9 and MMP-2 [16]. A novel fibrinolytic enzyme isolated from the earthworm Pheretima aspergillum can directly and strongly dissolve human thrombi and fibrin, and also activate human plasminogen to plasmin.

\section{Role of the Glycolipoprotein G-90}

G-90, a biologically active glycolipoprotein complex, isolated from whole dilong tissue extract (Eisenia foetida) [61]. There are several function attributed to G-90, including an insulin-like growth factor (IGF like), an immunoglobulinlike growth factor (IgFG-like) and epidermal growth factor (EGF) [48] and mitogenicity [72]. G-90 also has an apparent protective effect against the toxicity of $\mathrm{H}_{2} \mathrm{O}_{2}$ and stimulated the growth of the cell [73] and show anticoagulative and fibrinolytic activities [74, 75]. The results revealed that G-90 also participates in tissue regeneration and wound healing. The glycolipoprotein tissue homogenate extract from the earthworms Eisenia foetida (G-90) can activate signal transduction pathways, leading to wound healing. G90 promote and cause an increased concentration of EGF and FGF as observed $6 \mathrm{~h}$ after wounding on mouse skin [76].

Stimulation of cell proliferation during nerve regeneration usually involves initiation and progressive activity of growth factors. As mentioned previously, these growth factors (GDNF, BDNF, FGF and NGF) can activate MAPK pathway to stimulate Schwann cell migration. Highly expressed uPA in the epidermis of damaged tissue is regulated by the FGF-2 that activates MAPK kinase (MEKK-1) and its downstream ERK1/2 [49]. Despite these encouraging results, the possible beneficial effect of G-90 extracted from earthworm on peripheral nerve regeneration still remains unclear and requires further investigation and confirmation.

\section{Neural Cell Adhesion Molecule}

The neural cell adhesion molecule (NCAM) is a member of the immunoglobulin superfamily. Several studies showed that NCAM-induced neurite outgrowth depends on RasMAPK pathway activation [77]. NCAM-dependent cell migration to fibronectin required an intact MEK-ERK signaling pathway [78]. The putative adhesins of the immunoglobulin superfamily presumably from dilong extract could promote migration by MEK-ERK pathway activation. These bioactive compounds may indirectly cause ERK activation or directly activate plasminogen to plasmin by fibrinolytic enzyme, resulting in Schwann cell migration promotion during nerve regeneration.

\section{Action of Dilong Extract}

We suggest that dilong extract promotes proliferation by allowing Schwann cell survival. The IGF-IGFIR-Akt-Bcl2 axis stimulates tissue growth [21] and axonal regeneration [26]. IGF in certain cells, such as hematopoietic cells, functions as an inhibitor of cell death [79]. Activation of the PI3K/Akt pathway promotes cell survival. Activation of Akt leads to the phosphorylation of Bad [80] and connects a proximal survival signal with the Bcl-2 family to protect against apoptosis. Results of the immunoblotting assay showed that PI3K siRNA blocked the earthworm extractinduced expression of the anti-apoptotic proteins pBad and Bcl2. Cell cycle profiles were obtained for earthworm extracttreated Schwann cells after transfection with PI3K siRNA and for treated cells that were not transfected with PI3K siRNA. Knockdown of PI3K led to a significant inhibition of DNA synthesis in cells treated with earthworm extract for $24 \mathrm{~h}$ as evidenced by the fact that the number of $S$ phase proliferating cells decreased. These data indicate that Schwann cell survival and proliferation are PI3Kdependent processes that are mediated, atleast in part, by IGF-I. 


\section{The Future of Nerve Cell Regeneration and Possible Influence of Dilong}

The findings mentioned previously provide researchers another novel function during neuron regeneration. Although the treatment benefits of earthworms have been strongly supported, more precise studies are still required to: (i) reveal the precise and complete biochemical profile and (ii) suggest accurate doses of medication. In Boyd's study, the results suggested a dose-dependent facilitation and inhibition of peripheral nerve regeneration by brain-derived neurotrophic factor (BDNF). In contrast to the low-dose group, the high doses of BDNF (12-20 $\mathrm{g} \mathrm{day}^{-1}$ for 28 days) significantly inhibited motor axonal regeneration [81]. Chang's study suggested similar results [51]. As a word of caution, an excessive earthworm extract load in the medium could provoke an adverse response to recovery of neuron regeneration. In other words, there are threshold dosages above and below a certain effective dose. The findings of our study provide another novel function during neuron regeneration. However, the nerve growth-suppressing action by high doses of earthworm extract at concentrations of 250-1000 $\mathrm{mg} \mathrm{mL}^{-1}$, indicates that an excessive earthworm extract load in the medium could provoke an adverse response to neuron regeneration recovery. It demonstrates that excessive supplement could saturate the neurotrophin receptor, P75, to block the neuron regrowth-promoting function. Therefore, an appropriate dose of earthworm extract should be carefully selected to reach the highest potential for enhanced Schwann cell migration.

Based on these findings, we believe that certain components of earthworm extract can exert cell migration, proliferation and survival activity. The results demonstrate that dilong extract can stimulate Schwann cell migration and upregulate PAs and MMP2/9 expression mediated through the MAPK pathways, ERK1/2 and p38. Earthworm extract also stimulates Schwann cell proliferation and survival through the PI3K/Akt system mediated by IGF-I. The activity of dilong extract is probably related to its ability to induce $\mathrm{G}_{1}$ phase cell cycle progression by altering the expression of proteins that control the cell cycle (cyclin D1, cyclin E and cyclin A), resulting in the upregulation of the anti-apoptotic $\mathrm{Bcl}_{2}$ protein. Further analyses are essential to determine the presence of other bioactive compounds in dilong extract that might promote cell migration, survival and proliferation as well as the optimal dose of dilong extract.

\section{Funding}

This work was supported by grants from the China Medical University Hospital (DMR-96-047), 1PT Biotechnology Co., Ltd., and the China Medical University (CMU95-058, CMU95-060, CMU96-102 and CMU97-CMC-007) and supported in part by Taiwan Department of Health Clinical Trial and Research Center of Excellence (DOH99-TD-B-111-004).

\section{Acknowledgments}

The authors gratefully acknowledge the valuable insight and suggestions that were contributed by Professor Edwin
L. Cooper toward reviewing this manuscript. Y.-C. Cheng, C.-C. Lin, and C.-Y. Huang contributed equally to this work.

\section{References}

[1] R. P. Bunge, "Expanding roles for the Schwann cell: ensheathment, myelination, trophism and regeneration," Current Opinion in Neurobiology, vol. 3, no. 5, pp. 805-809, 1993.

[2] J. W. Fawcett and R. J. Keynes, "Peripheral nerve regeneration," Annual Review of Neuroscience, vol. 13, pp. 43-60, 1990.

[3] K. Torigoe, H.-F. Tanaka, A. Takahashi, A. Awaya, and K. Hashimoto, "Basic behavior of migratory Schwann cells in peripheral nerve regeneration," Experimental Neurology, vol. 137, no. 2, pp. 301-308, 1996.

[4] E. S. Anton, A. W. Sandrock Jr., and W. D. Matthew, "Merosin promotes neurite growth and Schwann cell migration in vitro and nerve regeneration in vivo: evidence using an antibody to merosin, ARM-1," Developmental Biology, vol. 164, no. 1, pp. 133-146, 1994.

[5] C. Ide, "Peripheral nerve regeneration," Neuroscience Research, vol. 25, no. 2, pp. 101-121, 1996.

[6] W. D. Snider, F.-Q. Zhou, J. Zhong, and A. Markus, "Signaling the pathway to regeneration," Neuron, vol. 35, no. 1, pp. 13-16, 2002.

[7] G. Pearson, F. Robinson, T. B. Gibson et al., "Mitogenactivated protein (MAP) kinase pathways: regulation and physiological functions," Endocrine Reviews, vol. 22, no. 2, pp. 153-183, 2001.

[8] S. Meintanis, D. Thomaidou, K. R. Jessen, R. Mirsky, and R. Matsas, "The neuron-glia signal $\beta$-neuregulin promotes Schwann cell motility via the MAPK pathway," GLIA, vol. 34, no. 1, pp. 39-51, 2001.

[9] P. P. Roux and J. Blenis, "ERK and p38 MAPK-activated protein kinases: a family of protein kinases with diverse biological functions," Microbiology and Molecular Biology Reviews, vol. 68, no. 2, pp. 320-344, 2004.

[10] C. Huang, K. Jacobson, and M. D. Schaller, "MAP kinases and cell migration," Journal of Cell Science, vol. 117, no. 20, pp. 4619-4628, 2004.

[11] K. B. Reddy, S. M. Nabha, and N. Atanaskova, "Role of MAP kinase in tumor progression and invasion," Cancer and Metastasis Reviews, vol. 22, no. 4, pp. 395-403, 2003.

[12] W. Ma and R. Quirion, "The ERK/MAPK pathway, as a target for the treatment of neuropathic pain," Expert Opinion on Therapeutic Targets, vol. 9, no. 4, pp. 699-713, 2005.

[13] P. Wiklund, P. A. Ekstrom, and A. Edstrom, "Mitogenactivated protein kinase inhibition reveals differences in signalling pathways activated by neurotrophin-3 and other growth-stimulating conditions of adult mouse dorsal root ganglia neurons," Journal of Neuroscience Research, vol. 67, no. 1, pp. 62-68, 2002.

[14] L. B. Siconolfi and N. W. Seeds, "Mice lacking tPA, uPA, or plasminogen genes showed delayed functional recovery after sciatic nerve crush," Journal of Neuroscience, vol. 21, no. 12, pp. 4348-4355, 2001.

[15] L. B. Siconolfi and N. W. Seeds, "Induction of the plasminogen activator system accompanies peripheral nerve regeneration after sciatic nerve crush," Journal of Neuroscience, vol. 21, no. 12, pp. 4336-4347, 2001.

[16] G. Murphy, S. Atkinson, R. Ward, J. Gavrilovic, and J. J. Reynolds, "The role of plasminogen activators in the regulation of connective tissue metalloproteinases," Annals of the New York Academy of Sciences, vol. 667, pp. 1-12, 1992. 
[17] L. B. Siconolfi and N. W. Seeds, "Mice lacking tissue plasminogen activator and urokinase plasminogen activator genes show attenuated matrix metalloproteases activity after sciatic nerve crush," Journal of Neuroscience Research, vol. 74, no. 3, pp. 430 434, 2003.

[18] S. Koochekpour, O. Sartor, M. Hiraiwa et al., "Saposin C stimulates growth and invasion, activates p42/44 and SAPK/JNK signaling pathways of MAPK and upregulates UPA/uPAR expression in prostate cancer and stromal cells," Asian Journal of Andrology, vol. 7, no. 2, pp. 147-158, 2005.

[19] M. Hecht, U. Heider, M. Kaiser, I. von Metzler, J. Sterz, and O. Sezer, "Osteoblasts promote migration and invasion of myeloma cells through upregulation of matrix metalloproteinases, urokinase plasminogen activator, hepatocyte growth factor and activation of p38 MAPK," British Journal of Haematology, vol. 138, no. 4, pp. 446-458, 2007.

[20] M. Schumacher, I. Jung-Testas, P. Robel, and E. E. Baulieu, "Insulin-like growth factor I: a mitogen for rat Schwann cells in the presence of elevated levels of cyclic AMP," Glia, vol. 8, no. 4, pp. 232-240, 1993.

[21] D. Le Roith and A. A. Butler, "Insulin-like growth factors in pediatric health and disease," Journal of Clinical Endocrinology and Metabolism, vol. 84, no. 12, pp. 4355-4361, 1999.

[22] E. DiCicco-Bloom and I. B. Black, "Insulin growth factors regulate the mitotic cycle in cultured rat sympathetic neuroblasts," Proceedings of the National Academy of Sciences of the United States of America, vol. 85, no. 11, pp. 4066-4070, 1988.

[23] Y. Aizenman and J. de Vellis, "Brain neurons develop in a serum and glial free environment: effects of transferrin, insulin, insulin-like growth factor-I and thyroid hormone on neuronal survival, growth and differentiation," Brain Research, vol. 406, no. 1-2, pp. 32-42, 1987.

[24] P. Caroni and P. Grandes, "Nerve sprouting in innervated adult skeletal muscle induced by exposure to elevated levels of insulin-like growth factors," Journal of Cell Biology, vol. 110, no. 4, pp. 1307-1317, 1990.

[25] C. L. Delaney, H.-L. Cheng, and E. L. Feldman, "Insulinlike growth factor-I prevents caspasemediated apoptosis in Schwann cells," Journal of Neurobiology, vol. 41, no. 4, pp. 540548, 1999.

[26] Y. Koriyama, K. Homma, K. Sugitani et al., "Upregulation of IGF-I in the goldfish retinal ganglion cells during the early stage of optic nerve regeneration," Neurochemistry International, vol. 50, no. 5, pp. 749-756, 2007.

[27] J. I. Jones and D. R. Clemmons, "Insulin-like growth factors and their binding proteins: biological actions," Endocrine Reviews, vol. 16, no. 1, pp. 3-34, 1995.

[28] G. Mairet-Coello, A. Tury, and E. DiCicco-Bloom, "Insulinlike growth factor-1 promotes G1/S cell cycle progression through bidirectional regulation of cyclins and cyclindependent kinase inhibitors via the phosphatidylinositol 3kinase/Akt pathway in developing rat cerebral cortex," Journal of Neuroscience, vol. 29, no. 3, pp. 775-788, 2009.

[29] G. M. Leinninger, C. Backus, M. D. Uhler, S. I. Lentz, and E. L. Feldman, "Phosphatidylinositol 3-kinase and Akt effectors mediate insulin-like growth factor-I neuroprotection in dorsal root ganglia neurons," FASEB Journal, vol. 18, no. 13, pp. 1544-1546, 2004.

[30] W. M. Campana, S. J. Darin, and J. S. O’Brien, "Phosphatidylinositol 3-kinase and Akt protein kinase mediate IGFI- and prosaptide-induced survival in Schwann cells," Journal of Neuroscience Research, vol. 57, no. 3, pp. 332-341, 1999.
[31] P. Maurel and J. L. Salzer, "Axonal regulation of Schwann cell proliferation and survival and the initial events of myelination requires PI 3-kinase activity," Journal of Neuroscience, vol. 20, no. 12, pp. 4635-4645, 2000.

[32] K. A. Sullivan, B. Kim, and E. L. Feldman, "Insulin-like growth factors in the peripheral nervous system," Endocrinology, vol. 149, no. 12, pp. 5963-5971, 2008.

[33] A. M. Vincent, B. C. Mobley, A. Hiller, and E. L. Feldman, "IGF-I prevents glutamate-induced motor neuron programmed cell death," Neurobiology of Disease, vol. 16, no. 2, pp. 407-416, 2004.

[34] C.-C. Tsai, M.-C. Lu, Y.-S. Chen, C.-H. Wu, and C.-C. Lin, "Locally administered nerve growth factor suppresses ginsenoside Rb 1-enhanced peripheral nerve regeneration," American Journal of Chinese Medicine, vol. 31, no. 5, pp. 665673, 2003.

[35] E. L. Cooper, B. Ru, and N. Weng, "Earthworms: sources of antimicrobial and anticancer molecules," Advances in Experimental Medicine and Biology, vol. 546, pp. 359-389, 2004.

[36] E. L. Cooper, "Complementary and alternative medicine, when Rigorous, can be science," Evidence-Based Complementary and Alternative Medicine, vol. 1, pp. 1-4, 2004.

[37] L. S. Ranganathan, Vermibiotechnology: From Soil Health to Human Health, Agrobios, 2006.

[38] C. L. Hsieh, T. H. Liu, J. G. Lin et al., "The relationship between Bu-Yang-Huan-Wu-Tang improve cerebral infarct and regulation of Nitric oxide in ischemia-reperfusion rats," Taiwanese Journal of Chinese Medicine, vol. 3, pp. 20-35, 2004.

[39] X. L. Wang, "Experimental research on Bu Yang Huan Wu Tang's effect on peripheral nerve regeneration," Journal of Shandong Medical University, vol. 37, pp. 41-43, 1999.

[40] Y. Y. Dong, "Experimental research on modified Bu Yang Huan Wu Tang to treat spinal cord injuries in rats," China Journal of Zheng Gu, vol. 10, pp. 3-4, 1998.

[41] E. L. Cooper, "CAM, eCAM, bioprospecting: the 21st century pyramid," Evidence-Based Complementary and Alternative Medicine, vol. 2, no. 2, pp. 125-127, 2005.

[42] M. Balamurugan, K. Parthasarathi, L. S. Ranganathan, and E. L. Cooper, "Hypothetical mode of action of earthworm extract with hepatoprotective and antioxidant properties," Journal of Zhejiang University: Science B, vol. 9, no. 2, pp. 141-147, 2008.

[43] M. Zhang, X. Li, Y. Liu, F. Ye, and G. Qiu, "Effects of extract of Dilong (Pheretima) on the scalded skin in rats," Journal of Traditional Chinese Medicine, vol. 26, no. 1, pp. 68-71, 2006.

[44] H. Mihara, M. Maruyama, and H. Sumi, "Novel thrombolytic therapy discovered from traditional oriental medicine using the earthworm," The Southeast Asian Journal of Tropical Medicine and Public Health, vol. 23, supplement 2, pp. 131140, 1992.

[45] S. Wei, P. Zhang, Y. Dang, H. Zhang, and B. Jiang, "Primary study on effect of various components of modified formula radix hedysari on peripheral nerve regeneration," Zhongguo Xiufu Chongjian Waike Zazhi, vol. 22, no. 9, pp. 1056-1059, 2008.

[46] S. Matsuba, "Complementary and alternative approaches to biomedicine," Evidence-Based Complementary and Alternative Medicine, vol. 1, pp. 345-348, 2004.

[47] W. Hu and T. Fu, "Isolation and properties of a novel fibrinolytic enzyme from an earth worm," Zhong Yao Cai, vol. 20, no. 2, pp. 78-81, 1997. 
[48] E. L. Cooper, T. M. Hrzenjak, and M. Grdiša, "Alternative sources of fibrinolytic, anticoagulative, antimicrobial and anticancer molecules," International Journal of Immunopathology and Pharmacology, vol. 17, no. 3, pp. 237-244, 2004.

[49] J. Witowsky, A. Abell, N. L. Johnson, G. L. Johnson, and B. D. Cuevas, "MEKK1 is required for inducible urokinasetype plasminogen activator expression," Journal of Biological Chemistry, vol. 278, no. 8, pp. 5941-5946, 2003.

[50] J. Yu, D. Bian, C. Mahanivong, R. K. Cheng, W. Zhou, and S. Huang, "P38 mitogen-activated protein kinase regulation of endothelial cell migration depends on urokinase plasminogen activator expression," Journal of Biological Chemistry, vol. 279, no. 48, pp. 50446-50454, 2004.

[51] Y. M. Chang, Y. T. Shih, Y. S. Chen et al., "Schwann cell migration induced by dilong extract via activation of PAs and MMP2/9 mediated through ERK1/2 and p38," Evidence-Based Complementary and Alternative Medicine. In press.

[52] R. N. Pittman and A. J. DiBenedetto, "PC12 cells overexpressing tissue plasminogen activator regenerate neurites to a greater extent and migrate faster than control cells in complex extracellular matrix," Journal of Neurochemistry, vol. 64, no. 2, pp. 566-575, 1995.

[53] E. Ulfhammer, P. Larsson, L. Karlsson et al., "TNF- $\alpha$ mediated suppression of tissue type plasminogen activator expression in vascular endothelial cells is NF- $\kappa \mathrm{B}$ - and p38 MAPKdependent," Journal of Thrombosis and Haemostasis, vol. 4, no. 8, pp. 1781-1789, 2006.

[54] C.-L. Hu, R. G. Cowan, R. M. Harman, and S. M. Quirk, "Cell cycle progression and activation of Akt kinase are required for insulin-like growth factor I-mediated suppression of apoptosis in granulosa cells," Molecular Endocrinology, vol. 18, no. 2, pp. 326-338, 2004.

[55] K. Homma, Y. Koriyama, K. Mawatari, Y. Higuchi, J. Kosaka, and S. Kato, "Early downregulation of IGF-I decides the fate of rat retinal ganglion cells after optic nerve injury," Neurochemistry International, vol. 50, no. 5, pp. 741-748, 2007.

[56] C. L. Delaney, H.-L. Cheng, and E. L. Feldman, "Insulinlike growth factor-I prevents caspasemediated apoptosis in Schwann cells," Journal of Neurobiology, vol. 41, no. 4, pp. 540548, 1999.

[57] K. D. Beck, L. Powell-Braxton, H.-R. Widmer, J. Valverde, and F. Hefti, "Igf1 gene disruption results in reduced brain size, CNS hypomyelination, and loss of hippocampal granule and striatal parvalbumin-containing neurons," Neuron, vol. 14, no. 4, pp. 717-730, 1995.

[58] C. M. Cheng, G. Joncas, R. R. Reinhardt et al., "Biochemicaland morphometric analyses show that myelination in the insulin-like growth factor 1 null brain is proportionate to its neuronal composition," Journal of Neuroscience, vol. 18, pp. 5673-5681, 1998.

[59] M. A. Stull, A. M. Rowzee, A. V. Loladze, and T. L. Wood, "Growth factor regulation of cell cycle progression in mammary epithelial cells," Journal of Mammary Gland Biology and Neoplasia, vol. 9, no. 1, pp. 15-26, 2004.

[60] Y. M. Chang, W. H. Kuo, T. Y. Lai et al., "RSC96 Schwann cell proliferation and survival induced by dilong extract through PI3K/Akt signaling mediated by IGF-I," EvidenceBased Complementary and Alternative Medicine. In press.

[61] T. Hrzenjak, M. Hrzenjak, V. Kasuba, P. EfenbergerMarinculic, and S. Levana, "A new source of biologically active compounds-earthworm tissue (Eisenia foetida, Lumbricus rubelus)," Comparative Biochemistry and Physiology A, vol. 102, no. 3, pp. 441-447, 1992.
[62] V. Ann Flørenes, G. Mari Mælandsmo, R. Faye, J. M. Nesland, and R. Holm, "Cyclin A expression in superficial spreading malignant melanomas correlates with clinical outcome," Journal of Pathology, vol. 195, no. 5, pp. 530-536, 2001.

[63] D. H. Walker and J. L. Maller, "Role for cyclin A in the dependence of mitosis on completion of DNA replication," Nature, vol. 354, no. 6351, pp. 314-317, 1991.

[64] D. R. Clemmons, J. J. van Wyk, and W. J. Pledger, "Sequential addition of platelet factor and plasma to BALB/c 3T3 fibroblast cultures stimulates somatomedin-C binding early in cell cycle," Proceedings of the National Academy of Sciences of the United States of America, vol. 77, pp. 6644-6648, 1980.

[65] N. E. Olashaw, J. J. van Wyk, and W. J. Pledger, "Control of late G0/G1 progression and protein modification by SmC/IGF I," American Journal of Physiology, vol. 253, no. 4, pp. C575-C579, 1987.

[66] M. A. Stull, M. M. Richert, A. V. Loladze, and T. L. Wood, "Requirement for IGF-I in epidermal growth factormediated cell cycle progression of mammary epithelial cells," Endocrinology, vol. 143, no. 5, pp. 1872-1879, 2002.

[67] Y. Tang, D. Liang, T. Jiang, J. Zhang, L. Gui, and W. Chang, "Crystal structure of earthworm fibrinolytic enzyme component A: revealing the structural determinants of its dual fibrinolytic activity," Journal of Molecular Biology, vol. 321, no. 1, pp. 57-68, 2002.

[68] J. Zhao, R. Pan, J. He, Y. Liu, D.-F. Li, and R.-Q. He, "Eisenia fetida protease-III-1 functions in both fibrinolysis and fibrogenesis," Journal of Biomedicine and Biotechnology, vol. 2007, Article ID 97654, 2007.

[69] D. Muir, "Metalloproteinase-dependent neurite outgrowth within a synthetic extracellular matrix is induced by nerve growth factor," Experimental Cell Research, vol. 210, no. 2, pp. 243-252, 1994.

[70] E. M. Muir, K. H. Adcock, D. A. Morgenstern et al., "Matrix metalloproteases and their inhibitors are produced by overlapping populations of activated astrocytes," Molecular Brain Research, vol. 100, no. 1-2, pp. 103-117, 2002.

[71] H. Nagase, "Activation mechanisms of matrix metalloproteinases," Biological Chemistry, vol. 378, no. 3-4, pp. 151-160, 1997.

[72] M. Hrzenjak, D. Kobrehel, S. Levanat, M. Jurin, and T. Hrzenjak, "Mitogenicity of the earthworm's (Eisenia foetida) insulinlike proteins," Comparative Biochemistry and Physiology B, vol. 104, no. 4, pp. 723-729, 1993.

[73] M. Grdisa, M. Popovic, and T. Hrzenjak, "Glycolipoprotein extract (G-90) from earthworm Eisenia foetida exerts some antioxidative activity," Comparative Biochemistry and Physiology A, vol. 128, no. 4, pp. 821-825, 2001.

[74] T. Hrženjak, M. Popović, T. Božić, M. Grdisa, D. Kobrehel, and L. Tiška-Rudman, "Fibrinolytic and anticoagulative activities from the earthworm Eisenia foetida," Comparative Biochemistry and Physiology B, vol. 119, no. 4, pp. 825-832, 1998.

[75] T. M. Hrženjak, M. Popović, and L. Tiška-Rudman, "Fibrinolytic activity of earthworms extract (G-90) on lysis of fibrin clots originated from the venous blood of patients with malignant tumors," Pathology and Oncology Research, vol. 4, no. 3, pp. 206-211, 1998.

[76] M. Grdiša, M. Popović, and T. Hrženjak, "Stimulation of growth factor synthesis in skin wounds using tissue extract (G-90) from the earthworm Eissenia foetida," Cell Biochemistry and Function, vol. 22, no. 6, pp. 373-378, 2004. 
[77] K. Kolkova, V. Novitskaya, N. Pedersen, V. Berezin, and E. Bock, "Neural cell adhesion molecule-stimulated neurite outgrowth depends on activation of protein kinase $\mathrm{C}$ and the Ras-mitogen-activated protein kinase pathway," Journal of Neuroscience, vol. 20, no. 6, pp. 2238-2246, 2000.

[78] S. Diestel, C. L. Hinkle, B. Schmitz, and P. F. Maness, "NCAM140 stimulates integrin-dependent cell migration by ectodomain shedding," Journal of Neurochemistry, vol. 95, no. 6, pp. 1777-1784, 2005.

[79] G. T. Williams, C. A. Smith, E. Spooncer, T. M. Dexter, and D. R. Taylor, "Haemopoietic colony stimulating factors promote cell survival by suppressing apoptosis," Nature, vol. 343, no. 6253, pp. 76-79, 1990.

[80] S. R. Datta, H. Dudek, T. Xu et al., "Akt phosphorylation of BAD couples survival signals to the cell- intrinsic death machinery," Cell, vol. 91, no. 2, pp. 231-241, 1997.

[81] J. G. Boyd and T. Gordon, "A dose-dependent facilitation and inhibition of peripheral nerve regeneration by brain-derived neurotrophic factor," European Journal of Neuroscience, vol. 15, no. 4, pp. 613-626, 2002. 


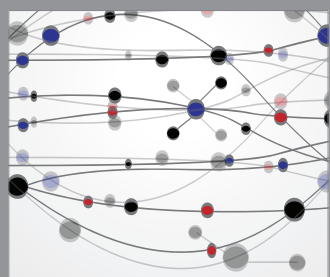

The Scientific World Journal
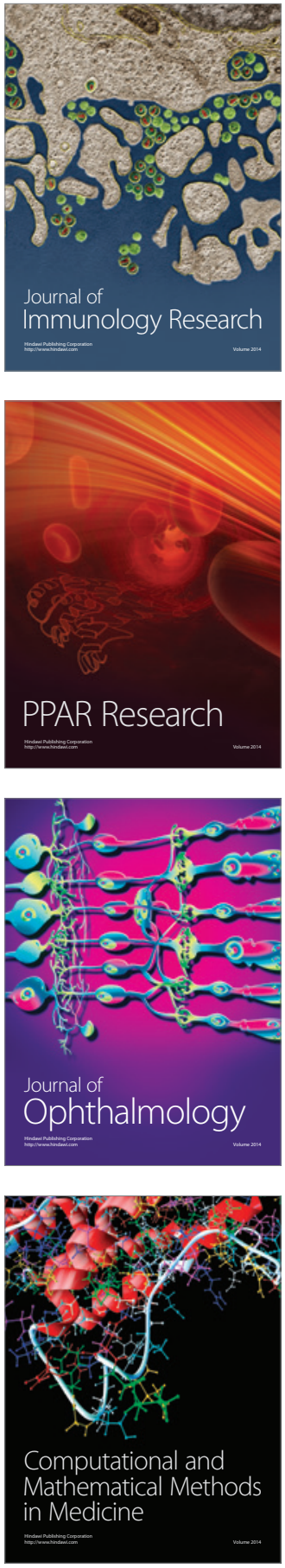

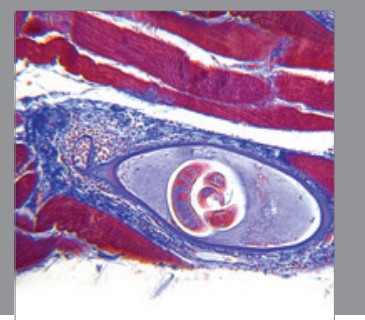

Gastroenterology

Research and Practice
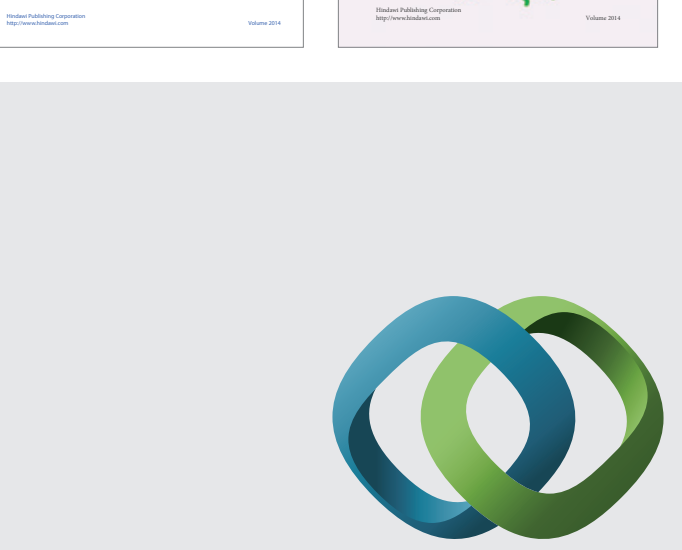

\section{Hindawi}

Submit your manuscripts at

http://www.hindawi.com
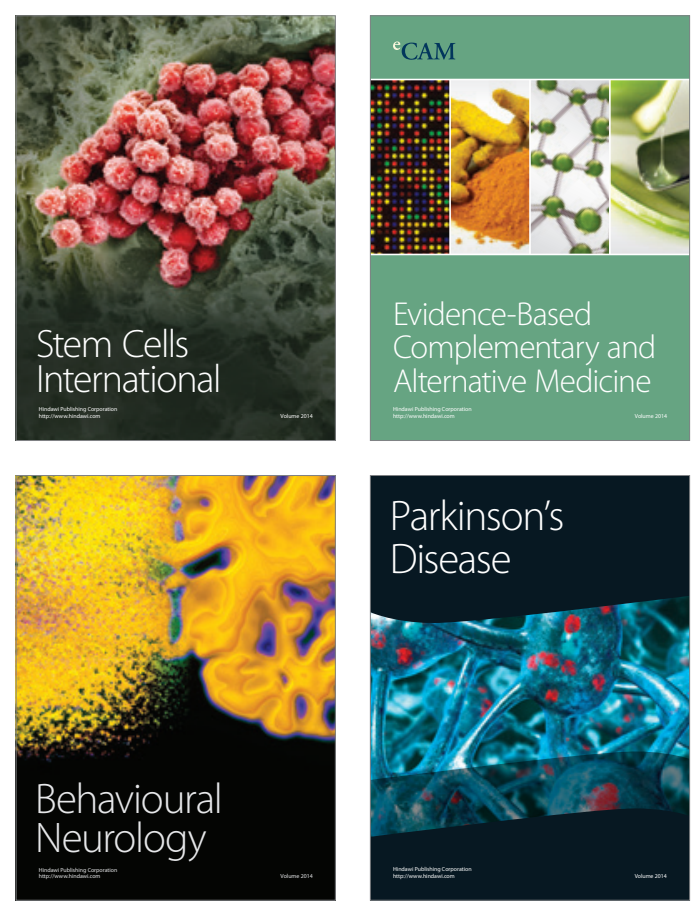

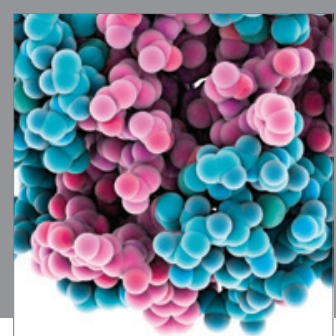

Journal of
Diabetes Research

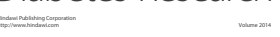

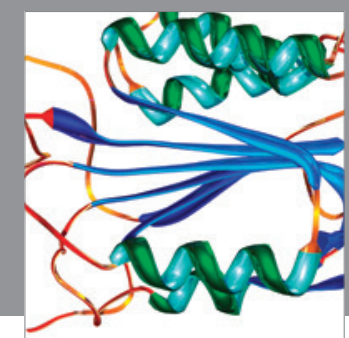

Disease Markers
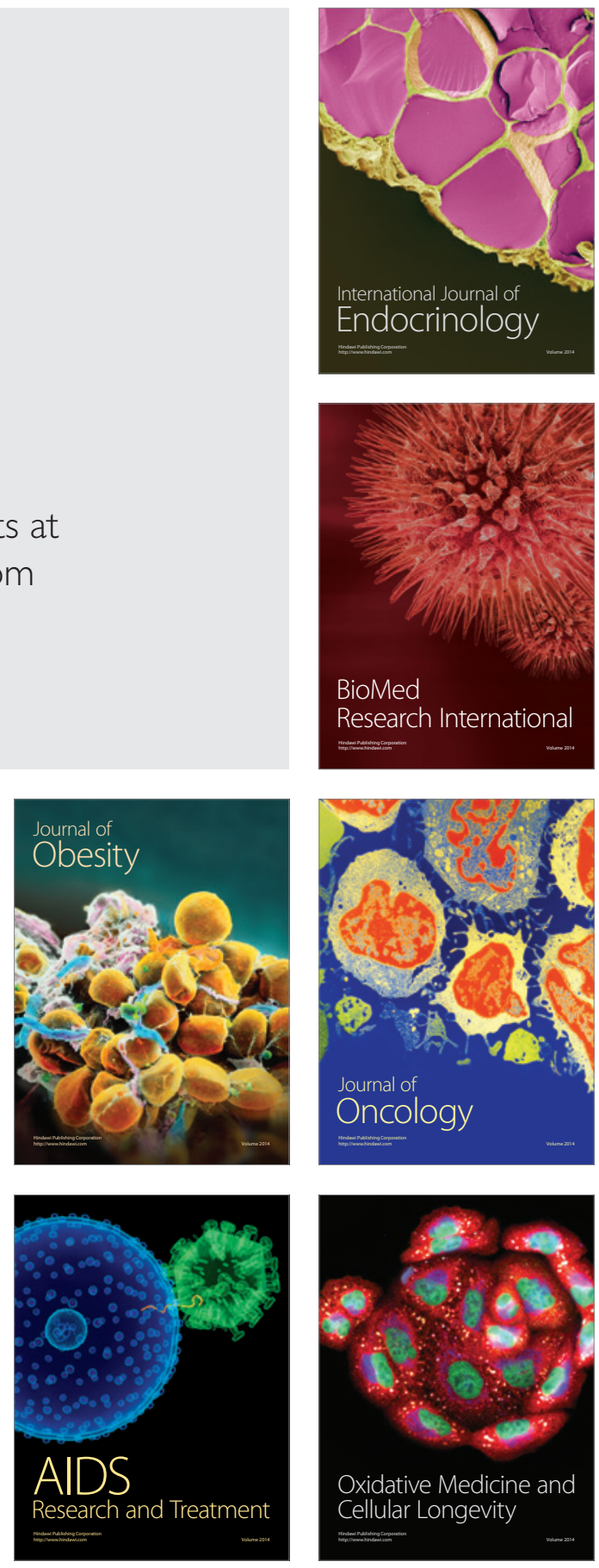